\title{
A Collaborative Aesthetic-driven Virtual Fitness Game
}

\author{
Lizhen $\operatorname{Han}^{1,2}$, Mingmin Zhang ${ }^{1}$, Feng Tian ${ }^{3}$, and Zhigeng $\operatorname{Pan}^{4} \bowtie$ \\ ${ }^{1}$ Zhejiang University, Hangzhou, China \\ ${ }^{2}$ Shandong University of Arts, Jinan, China \\ ${ }^{3}$ Bournemouth University, Bournemouth BH, UK \\ ${ }^{4}$ Hangzhou Normal University, Hangzhou, China \\ hlz-1@126.com, \\ zhangmm95ezju.edu.cn, \\ ftian@bournemouth.ac.uk, \\ zgpan@hznu.edu.cn四
}

\begin{abstract}
By introducing a collaborative aesthetic-driven virtual fitness game, we give a possible solution to guide and encourage people who have sedentary lifestyle to do regular physical activity with enough intensity, duration and frequency. This method bridges physical interaction with a stationary bicycle and visual changes in a virtual environment, and combines it with a multiplayer game. During the test of game playing, individual participant's effort to meet their own target heart rate zone contributes to a team effort of aesthetic immersion overall.
\end{abstract}

Keywords: aesthetic-driven interaction, multiplayer collaboration, aesthetic immersion, real-time bio-feedback, virtual fitness game

\section{Introduction}

Doing regular physical activity with enough amounts of intensity, duration and frequency is a healthy lifestyle. Helping people to form such a healthy lifestyle is a hot and challenging area in the fields of computer aided exergame design.

Aesthetic pleasure is a kind of powerful affective experience. It can enhance immersion and engagement of virtual reality environment. Based on this, we present a collaborative aesthetic-driven virtual fitness game as a low cost while pervasive solution to encourage effective fitness training. In this game, the aesthetic visual effects of a virtual world are tightly associated with the player's real time heart rate level, while the heart rate level reflects the subject's physiological and psychological

adfa, p. 1, 2011

C Springer-Verlag Berlin Heidelberg 2011 
state accordingly. By physically acting on the control of the representation of one component part of the virtual world, players will be engaged to cooperate on the creation of aesthetically pleasing virtual surroundings, meanwhile, to immerse themselves into target fitness training zones. A pilot in-lab user study was conducted to evaluate the effectiveness and enjoyment of the game. We find that this kind of persuasive technology is helpful on encouraging effective exercise training.

The main contribution of this work lies in: 1 . put forward a new idea for exergame design - the multiplayer game with the aesthetic experience act as the cooperation strategy and goal; 2 . complete a prototype system to demonstrate this aesthetic cooperation method.

The rest of the paper is organized as follows. In Section 2, we give a general review of related works. Then, we describe our method of aesthetic cooperation and illustrate the game design in details in Section 3. Finally, in Section 4, general conclusions are drawn.

\section{$2 \quad$ Related Works}

Our previous studies $[1,2,3]$ have found that combine people's instinctive pursuit of beauty with their real-time bio-feedback and immersive somatosensory interaction into one well designed system, can help people to be happy to carry out regular and effective exercise. In such a system, individual players tend to perform better to stay in a relatively beautiful virtual world, rather than in a relatively ugly world. So, what is the player's performance in a collaborative multiplayer mode? This is what we want to communicate in this research.

\subsection{Exergame for Fitness Training}

Exergames are regarded as a possible solution of encouraging more physical activity in a funny video game play way $[4,5,6,7]$. To encourage players to do more physical activity, some exergames aim to capture the player's action, emotion or the physiological data such as heart rate to control the gameplay $[8,9$,$] or to auto-adapt the game level for fair$ game play experiences $[10,11,12]$. Some try to constrain the player for more quantity and intensity by setting higher objectives or goals $[13,14]$. 
Others use competitions or award strategies to lure player spending more time [15].

Most of the approaches above adopt compulsive incentive strategies with constraints to attract and retain players. Through seemingly endless challenges, attractive stories, definite tasks, clear targets, excited competitions, honorary medals, etc., players are spurred to try their best to win. However, fitness training is not simply the faster the better. Exerciser may need to maintain their heart rate at a target level. Sinclair et al. [16] advocate that exergame should take the guidelines of American College of Sports Medicine as a scientific guarantee of true health, but few exergame abided it strictly.

\subsection{Aesthetical Immersion in Virtual Reality}

Immersion is vital for virtual reality system. It provides realistic experience, even though the virtual world may not simulate an actual realworld location. Using aesthetical immersion as incentive strategy for game play has not been explored in virtual reality application before.

Growing awareness has noticed that aesthetic design is a key component of usability [17], and a decisive aspect for marketplace success [18]. More and more researchers are exploring the potential usability of aesthetics on designing interactive systems. These researches can be divided into two directions. One takes beauty as an ornament for giving system more visual and emotional attraction [19]. Aesthetic aspects are most like an added bonus of the system and relatively independent from the system itself. Another one is the pragmatist approach, which looks aesthetics attributes from its practical and functional aspect. The representative work is the concept of aesthetic interaction [20].

Although the two directions of form and function are definite, researchers still struggling on come up with a methodological approach to the subjective concept of aesthetics, having typically concerned itself with more scientific methods [21].

\section{$3 \quad$ Method}

Aiming to be available for the public as much as possible, we choose the economy and pervasive technology to get basic immersion of virtual 
game scene. Figure 1 illustrate our method in the system implementation level. The use of advanced virtual reality devices than the use of this basic one can provide more exciting experience.

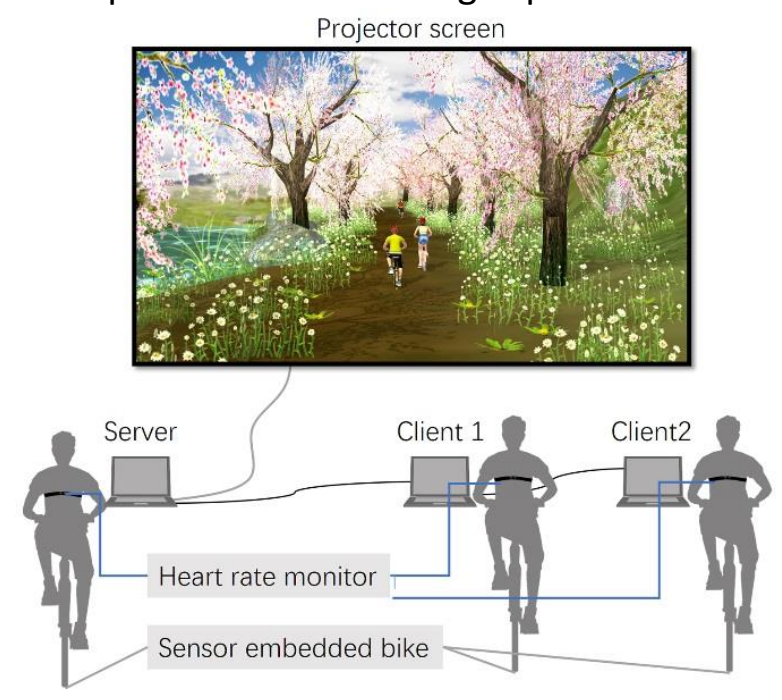

Fig. 1. Illustration of our system implementation.

In the multiplayer model, there are one server and multiple clients working together. Each player put on a wireless heart rate monitor and cycling on a sensor embedded bicycle. Her/his heart rate level, i.e. one's exercise intensity, is visualized as an element of a virtual scene. This element is selected from a library at the beginning of the gameplay by the player. The speed and direction of the player's bike are used to control the movement of their virtual avatar. By this way, during cycling, a virtual landscape which is composed of multiplayer's heart rate visualizations will be generated timely (Figure 2). The strength of this game entry is the hands-on, physical interaction which feeds into a virtual experience.

Totally there are five typical states of each element, which correspond to five exercise training zones reasonably, including aerobic zone, anaerobic zone and VO2 Max zone. The virtual scene can be composed of several elements. Each element is being controlled by one player. Thus, variety of combination effects will be presented under the collaboration of each player's performance. Adding the extra dimension of 
multiple players contributing their physical effort together adds an interesting layer of complexity.

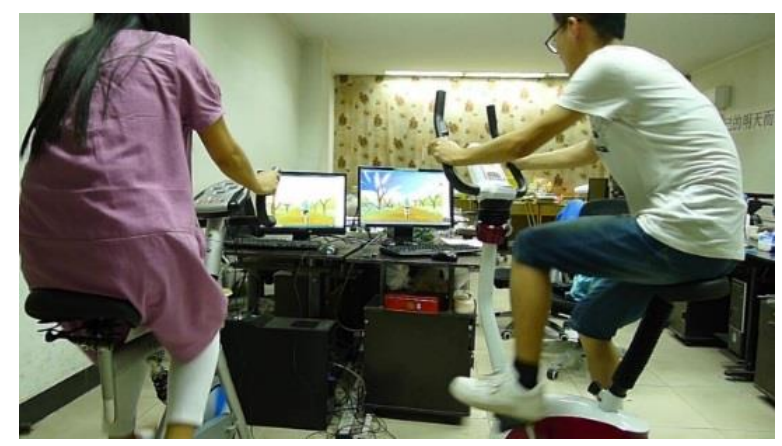

Fig. 2. Two players are playing our game with two sets of equipment. Wireless heart-rate monitors are worn on their chest.

According to the gestalt theory of visual perception [22], aesthetic pleasure is often results from the harmony of the parts of the whole, and from the balance of the underlying driven force as well as the tension reducing. On the contrary, ugly often means disharmony, full of tension and out of balance, which leads to the feelings of uncomfortable. Accordingly, the variety of collaborative virtual scenes can give rise to different aesthetic emotion, from disgust to happy, as shown in Figure 3. The more beautiful and vigorous the appearance of the virtual environment, the healthier and livelier each player's body status becomes and, the more emotional pleasure and immersive feeling the groups experiences.

Human pursue for beauty and avoid of ugly instinctively. They are more tolerant to stay in a beautiful environment rather than in an uncomfortable one. So, will all the players work together for the goal of creating a beautiful world which can be roamed and shared? Furthermore, do they really get the benefits of effective exercise by keeping their physical activity at the target exercise training zone? The results of a pilot in-lab user study ( 8 male and 4 females, graduate students, two or three persons one group, 15 minutes per ride, three times per person, random grouping each time) gave a positive response. 

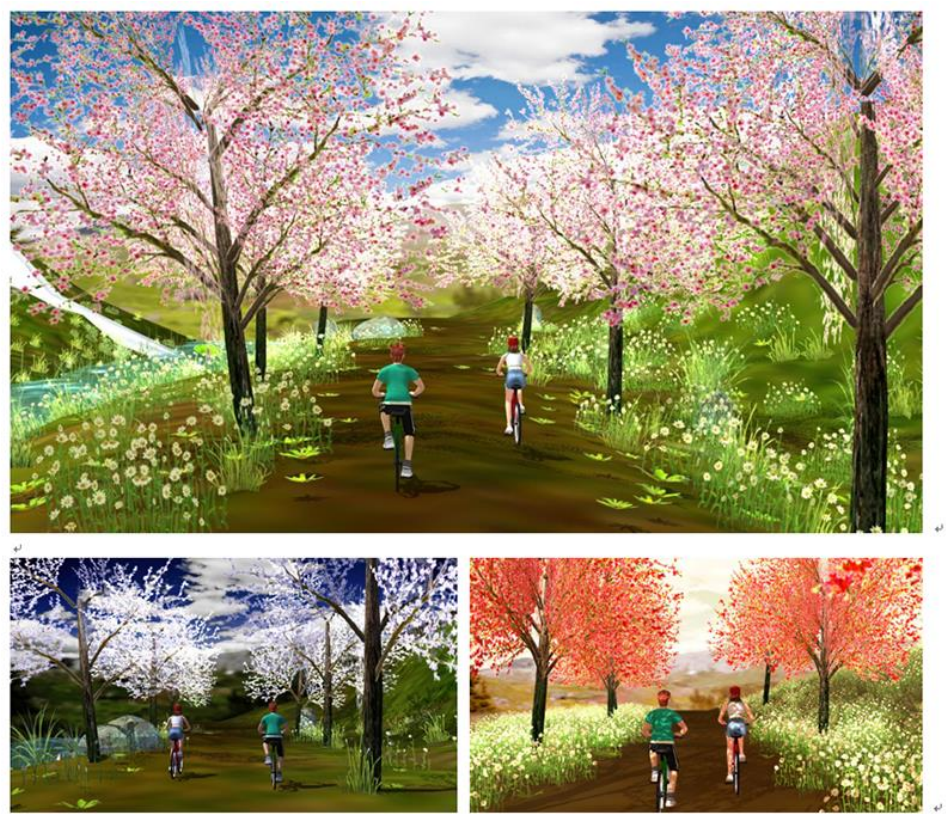

a) Harmony but different in aesthetic degree. Harmony means the players are in the same heart rate level at the same time. Different aesthetic degree means different body state and exercise intensity. .
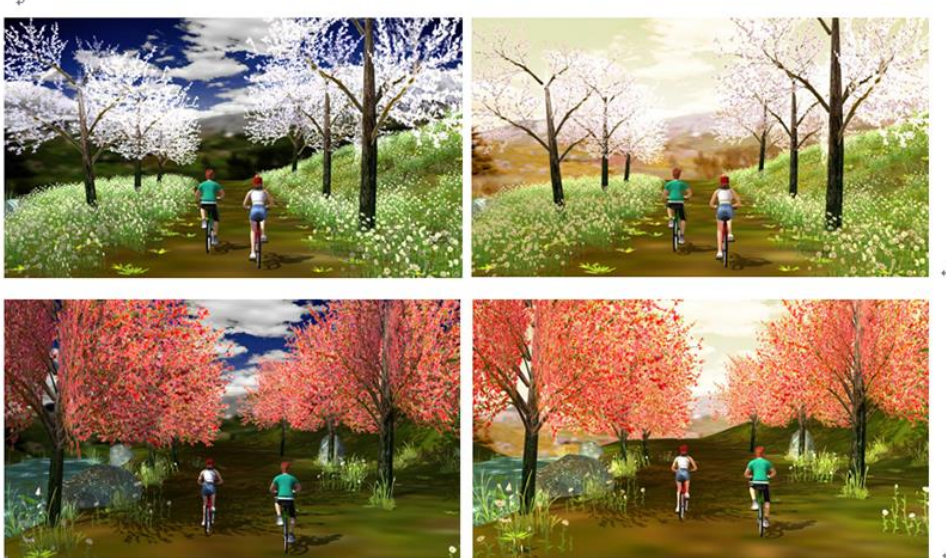

b) Disharmony, less beautiful. These picture indicate that the game players are not uniform in the exercise intensity. Disharmony leads to uncomfortable feelings of ugly. .

Fig. 3. Illustration of some typical collaborative scenes in a two-player case. The two players could see each other nearby means they have the similar speed at the same place. 


\section{Conclusion}

We address an interesting position on improving the interaction of a virtual world through aesthetic cooperation. Physiological measures which are coupled to the visual aspects of the game give guarantee to the effectiveness and individuality.

Whether it is in the public display space, or in the personal home environment, our virtual fitness game could be attractive. The reason lies in the followings: 1) Innovation. The use of biometrics to adjust the aesthetic sense of harmony in the scene is unique; 2 ) Collaboration. The concept of cooperation on create beauty as well as to maintain health is interesting. It will also push the boundaries of artistic creativity; 3 ) Interaction. The control of the game is natural, free and real-time; 4) Immersion. Somatosensory interaction and aesthetic pleasure will enhance the immersion of virtual reality; 5) Personality. The collaborative generated virtual world is unique and full of player's personal information of their inner body state. This is amazing.

We hope our work of aesthetically cooperation, can bring some inspiration to designers on how to develop exertion game or training system. In the future, in-depth user study is needed to evaluate the game design, a quantitative and qualitative analyses of its results is necessary for drawing a most precise conclusion. We also plan to enrich the elements library to give more creative freedom for the players. The quality of virtual scene's appearance will also be strengthened.

\section{References}

1. Lizhen Han, Mingmin Zhang, Feng Tian, AND Xinting Wang (2015) Heart-Creates-Worlds: An Aesthetic Driven Fitness Training System. Image and Graphics. Springer International Publishing, 599-608.

2. Lizhen Han, Mingmin Zhang, Zhigeng Pan, Qingru Meng, AND Feng Tian (2014) Aestheticdriven interaction: a pleasurable persuasive technology for health behavior change. SIGGRAPH ASIA Posters 2014, ACM, 15.

3. Mingmin Zhang, Mingliang Xu, Lizhen Han, Yong Liu, Pei Lv, AND Gaoqi He (2012) Virtual Network Marathon with immersion, scientificalness, competitiveness, adaptability and learn-ing. Computers \& Graphics 36(3), 185-192.

4. Sinclair, J., Hingston, P., \&Masek, M. (2007) Considerations for the design of exergames. In Pro-ceedings of the 5th international conference on Computer graphics and interactive tech-niques in Australia and Southeast Asia (pp. 289-295).ACM. 
5. Baranowski, T., Buday, R., Thompson, D. I., \&Baranowski, J. 2008. Playing for real: video games and stories for health-related behavior change. American journal of preventive medicine, 34(1), 74-82.

6. Berkovsky, S., Coombe, M., Freyne, J., Bhandari, D., \&Baghaei, N. 2010. Physical activity motivating games: virtual rewards for real activity. In Proceedings of the SIGCHI Conference on Human Factors in Computing Systems, pp. 243-252. ACM.

7. Yim, J., \& C. Nicholas Graham, T. 2007. Using games to increase exercise motivation. Future Play '07 Proceedings of the 2007 conference on Future Play.

8. Hamilton, I., Imperatore, G., Dunlop, M. D., Rowe, D., \& Hewitt, A. 2012. Walk2Build: a GPS game for mobile exergaming with city visualization. In Proceedings of the 14 th international conference on Human-computer interaction with mobile devices and services companion, pp. 17-22. ACM.

9. Lane N D, Lin M, Mohammod M, et al. BeWell: Sensing Sleep, Physical Activities and Social Interactions to Promote Wellbeing. 2014. Mobile Networks and Applications, 19(3):345-359.

10. Park T, Hwang I, Lee $U$, et al. 2012. ExerLink: enabling pervasive social exergames with heterogeneous exercise devices[J]. 10th International conference on Mobile systems, applications, and services.

11. Buttussi, F., Chittaro, L., Ranon, R., \& Verona, A. 2007. Adaptation of graphics and gameplay in fitness games by exploiting motion and physiological sensors. In Smart Graphics. pp. 85-96. Springer Berlin Heidelberg.

12. Lamoth, C. J., Alingh, R., \& Caljouw, S. R. 2012. Exergaming for elderly: Effects of different types of game feedback on performance of a balance task. Stud Health Technol Inform, $181,103-107$.

13. Masuko, S., \& Hoshino, J. 2006. A fitness game reflecting heart rate. In Proceedings of the 2006 ACM SIGCHI international conference on Advances in computer entertainment technology, p. 53. ACM.

14. Doyle, J., Kelly, D., Patterson, M., \& Caulfield, B. 2011. The effects of visual feedback in therapeutic exergaming on motor task accuracy. In Virtual Rehabilitation (ICVR), 2011 International Conference on, pp. 1-5. IEEE.

15. Diakopoulos, N., Kivran-Swaine, F., \& Naaman, M. (2011). Playable data: characterizing the design space of game-y infographics. In Proceedings of the SIGCHI Conference on Human Factors in Computing Systems (pp. 1717-1726). ACM.

16. Sinclair, J., Hingston, P., Masek, M., \& Nosaka, K. K. (2009). Using a virtual body to aid in exergaming system development. IEEE computer graphics and applications, (2), 39-48.

17. Norman, D. A. (2007). Emotional design: Why we love (or hate) everyday things. Basic books.

18. Bloch, P. H. (1995). Seeking the ideal form: product design and consumer response. The Journal of Marketing, 16-29.

19. FOGARTY, J, F. J., AND HUDSON, S. E.. Aesthetic Information Collages: Generating Decorative Displays that Contain Information. 2001,141-150.

20. TRACTINSKY, N. 2004. Toward the Study of Aesthetics in Information Technology. In International Conference on Information Systems, 771-780.

21. JUNG, H., A. Y. L., AND BARDZELL, J. SKIN: dDesigning aAesthetic ilnteractive sSurfaces. In TEI '10 Proceedings of the fourth international conference on Tangible, embedded, and embodied interaction, 2010, 85-92.

22. Arnheim R., 1969. Visual Thinking. Berkeley: University of California Press. 